University of Wollongong

Research Online

2020

Effects of nano-clay content, foaming temperature and foaming time on density and cell size of PVC matrix foam by presented Least Absolute Shrinkage and Selection Operator statistical regression via suitable experiments as a function of MMT content

\author{
Zhixiong Li \\ University of Wollongong, lizhixio@uow.edu.au \\ Hamzeh Shahrajabian \\ Seyed Amin Bagherzadeh \\ Hamid Jadidi \\ Arash Karimipour
}

See next page for additional authors

Follow this and additional works at: https://ro.uow.edu.au/eispapers1

Part of the Engineering Commons, and the Science and Technology Studies Commons 


\title{
Effects of nano-clay content, foaming temperature and foaming time on density and cell size of PVC matrix foam by presented Least Absolute Shrinkage and Selection Operator statistical regression via suitable experiments as a function of MMT content
}

\begin{abstract}
Present article aims to investigate the effect of nano-clay content, foaming temperature and foaming time on the density and cell size of the PVC matrix foam. The cell size would affect the insulating and mechanical properties. The foaming temperature is set in three levels of 70,80 and $90^{\circ} \mathrm{C}$, foaming time is set in three levels of 10,20 , and $30 \mathrm{~s}$; and nano-clay is in content of 1,3 , and $5 \mathrm{wt} \%$. Outputs consist the density and cell size, which affect impact the thermal conductivity, mechanical properties and the weight of the polymer foam. In addition the Least Absolute Shrinkage and Selection Operator (LASSO) regression method is employed in order to improve both the precision and generalization of the estimated foam density and cell size as functions of the MMT content, the foaming temperature and the foaming time. LASSO is found a suitable approach to predict the sample properties.
\end{abstract}

\section{Keywords}

cell, size, pvc, matrix, foam, presented, least, absolute, shrinkage, selection, operator, statistical, regression, via, suitable, experiments, function, $\mathrm{mmt}$, density, content, effects, nano-clay, content, temperature, time, foaming

Disciplines

Engineering | Science and Technology Studies

\section{Publication Details}

Li, Z., Shahrajabian, H., Bagherzadeh, S. Amin., Jadidi, H., Karimipour, A. \& Tlili, I. (2020). Effects of nanoclay content, foaming temperature and foaming time on density and cell size of PVC matrix foam by presented Least Absolute Shrinkage and Selection Operator statistical regression via suitable experiments as a function of MMT content. Physica A: Statistical Mechanics and its Applications, 537 122637-1-122637-11.

\section{Authors}

Zhixiong Li, Hamzeh Shahrajabian, Seyed Amin Bagherzadeh, Hamid Jadidi, Arash Karimipour, and Iskander Tlili 
Effects of nano-clay content, foaming temperature and foaming time on density and cell size of PVC matrix foam by presented Least Absolute Shrinkage and Selection Operator statistical regression via suitable experiments as a function of MMT content

\title{
Zhixiong Li ${ }^{1,2}$, Hamzeh Shahrajabian³, Seyed Amin Bagherzadeh ${ }^{3}$,
} Hamid Jadidi ${ }^{3}$, Arash Karimipour ${ }^{3}$, Iskander Tlili ${ }^{4,5, *}$

${ }^{1}$ Ocean College, Minjiang University, Fuzhou 350108, China.

${ }^{2}$ State Key Laboratory of Hydraulic Engineering Simulation and Safety, Tianjin University, Tianjin 300350, China.

${ }^{3}$ Department of Mechanical Engineering, Najafabad Branch, Islamic Azad University, Najafabad, Iran.

${ }^{4}$ Department for Management of Science and Technology Development, Ton Duc Thang University, Ho Chi Minh City, Vietnam.

${ }^{5}$ Faculty of Applied Sciences, Ton Duc Thang University, Ho Chi Minh City, Vietnam.

* Corresponding author; Email: iskander.tlili@tdtu.edu.vn

\begin{abstract}
Present article aims to investigate the effect of nano-clay content, foaming temperature and foaming time on the density and cell size of the PVC matrix foam. The cell size would affect the insulating and mechanical properties. The foaming temperature is set in three levels of 70, 80 and $90{ }^{\circ} \mathrm{C}$, foaming time is set in three levels of 10, 20, and 30 seconds; and nano-clay is in content of 1,3 , and 5 wt.\%. Outputs consist the density and cell size, which affect impact the thermal conductivity, mechanical properties and the weight of the polymer foam. In addition the Least Absolute Shrinkage and Selection Operator (LASSO) regression method is employed in order to improve both the precision and generalization of the estimated foam density and cell size as functions of the MMT content, the foaming temperature and the foaming time. LASSO is found a suitable approach to predict the sample properties.
\end{abstract}

\section{Keywords:}

Least Absolute Shrinkage \& Selection Operator; nano-clay content; foaming temperature; density and cell size; PVC matrix foam 


\section{Introduction}

The thermal conductivity is an important characteristic of polymer foam that is very interesting in insulation applications in various industries. The thermal conductivity is affected by the cell size and the density of the polymer foam. By decreasing the cell size to the micron size (under $100 \mu \mathrm{m})$, the thermal conductivity decreases dramatically [1,2], and leads to manufacture the microcellular foam as an efficient insulation material [3]. Sriharsha et al. investigated the effect of cell size on thermal conductivity by molecular dynamics and finite element, and concluded that thermal conductivity depends on cell size strongly [4].

There is various method to produce polymer foam, but the mass method one of the most effective way to fabricate polymer microcellular foam $[5,6]$. In this method, the inert gas (nitrogen or carbon dioxide) is used as the foaming agent [7-9]. In the microcellular foam, when the cell size is lower than critical cracks, the density can be decreased without losing significant mechanical properties. The mass method consists generally of three steps: 1- gas adsorption, 2- nucleation, and 3- cell growth. In the first step, the sample is suspended in high pressure gas vessel to penetrate the gas molecules into the sample to saturate the sample. Then, the sample is immerged in hot glycerin with a specific temperature (foaming temperature) at a specified time (foaming time) to growth the cells. Finally, the sample is floated in cold water to prevent excessive cell growth.

The insulating properties which affected by cell size and weight (density) two important factors in polymer foams, hence manufacturing the foams possesses low cell size and density is necessary. nano-fillers are effective materials that are used in polymer foams to affect the density and cell size $[10,11]$. The nano-fillers causes to increase the density by increasing the nucleation sites, to decrease the cell size by preventing the excessive cell growth $[12,13]$, and to produce homogenous cell size $[14,15]$. An interesting nano-filler which is used by many researchers in polymer foam to produce the foam with suitable cell size and density is nano clay [16-18]. Based on previous researches the weight percentage of the nano-clay is an effective parameter for the cell size and the density [19-33].

Considering the importance of the thermal conductivity, mechanical properties and weight of polymer foam, it is necessary to find the relationship between input parameters of the foaming process, and the cell size and density. In mass method, the foaming time and foaming temperature are effective on cell size and density. 
The Least Absolute Shrinkage and Selection Operator (LASSO) regression method is employed in order to improve both the precision and generalization of the estimated foam density and cell size as functions of the MMT content, the foaming temperature and the foaming time.

\section{Problem statement}

The purpose of this research is to study the effect of nano-clay content, foaming temperature and foaming time on the density and cell size of the PVC matrix foam. The cell size affects insulating and mechanical properties. The foaming temperature was set in three levels of 70, 80 and $90{ }^{\circ} \mathrm{C}$, foaming time was set in three levels of 10, 20, and 30 seconds; and nano-clay was in content of 1,3 , and $5 \mathrm{wt} . \%$. Outputs consist the density and cell size, which affect impact the thermal conductivity, mechanical properties and the weight of the polymer foam.

\section{Experimental}

\subsection{Material}

The PVC with commercial grade of S6558 as the matrix of polymer foam was purchased from PGP company. Commercial grades of AIM, and AZ-355, were used as stabilizer and impact modifier respectively. The calcium stearate and stearic acid were used as the lubricants. Table 1 presents the PVC ingredient. The Montmorillonite modified with a quaternary ammonium salt purchased from Southeren Clay Products (USA).

Table 1- The ingredient of PVC.

\begin{tabular}{|l|c|}
\hline Material & Amount (phr) \\
\hline The powder of PVC & 100 \\
\hline Impact modifier & 5 \\
\hline Stearic acid & 0.5 \\
\hline Stabilizer & 5 \\
\hline Calcium stearate & 1 \\
\hline
\end{tabular}




\subsection{Sample preparation}

All oaf materials (polymer matrix and filler) were dried in an oven at temperature of $80{ }^{\circ} \mathrm{C}$ for $24 \mathrm{~h}$. The samples were prepared by internal mixer at the temperature of $160160{ }^{\circ} \mathrm{C}$ time of 6 min, and screw revolution of $60 \mathrm{rpm}$. The nano-clay was added into PVC matrix in content of 1,3 , and 5 wt. $\%$. The output of the internal mixer was converted into granules. The nanocomposite granules were converted into the sheets with $1 \mathrm{~mm}$ thickness by hot press technique at pressure of $50 \mathrm{MPa}$, temperature of $175^{\circ} \mathrm{C}$ for $50 \mathrm{~min}$. Finally, the sheets were cut in $2 \mathrm{~mm}$ width.

\subsection{Foaming the sample}

Foaming the solid nanocomposites samples were done by mass method. The sample suspended in the $\mathrm{CO}_{2}$ vessel at high pressure of $4.5 \mathrm{MPa}$, temperature of $50{ }^{\circ} \mathrm{C}$ for $45 \mathrm{~h}$ to penetrate the $\mathrm{CO}_{2}$ molecules into solid sample, and the sample is saturated with gas. Then the sample was floated at hot glycerin at specific temperature $\left(60-80{ }^{\circ} \mathrm{C}\right)$, and specific time (2040 s) to create the air cells. The samples immediately were immersed in water containing the ice to prevent excess cell growth and gas escape.

\subsection{Design variables (Input parameters)}

The effective parameters which affect the foaming conditions of the nanocomposite samples are foaming temperature $(\mathrm{T})$, foaming time $(\mathrm{t})$, and nano-clay content $(\varphi)$. These three parameter were considered as the input parameter. The table 2 present the parameter levels.

Table 2- Levels of variables.

\begin{tabular}{|l|c|c|c|}
\hline Variable, code & Level 1 & Level 2 & Level 3 \\
\hline MMT content, $\Phi$ (wt. \%) & 1 & 3 & 5 \\
\hline $\begin{array}{l}\text { Foaming temperature, T } \\
\left.\mathbf{l}^{\circ} \mathbf{C}\right)\end{array}$ & 60 & 70 & 80 \\
\hline Foaming time, $\mathbf{t}$ (s) & 20 & 30 & 40 \\
\hline
\end{tabular}




\subsection{Response (Output parameters)}

The foam density $(\rho)$ and cell size (A) have important features in the selection and use of polymer foams. In this study, the density and cell size were selected as the responses (output parameters). The density of the samples was measured by Archimedes method. To determine the cell size, at first, the samples were broken in liquid nitrogen, and the SEM (Scanning electron microscopy) images were taken from the fracture surface of the samples. Then, the cell size of the samples was determine by image processing software MIP4. Figure 1 shows the cell size measurement by MIP4 software.

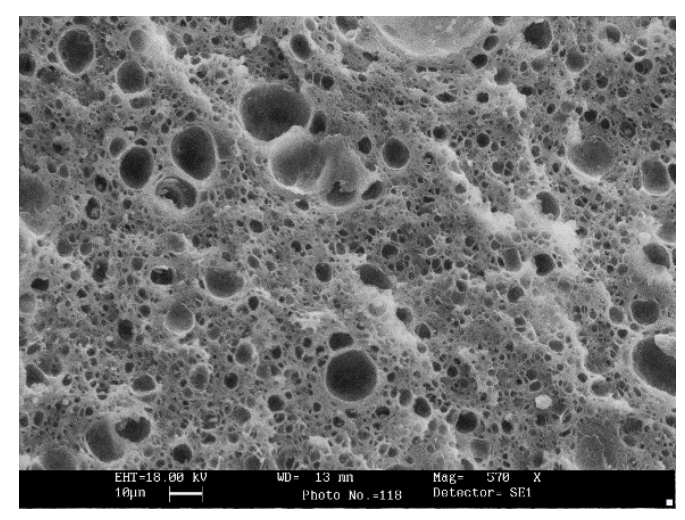

(a)

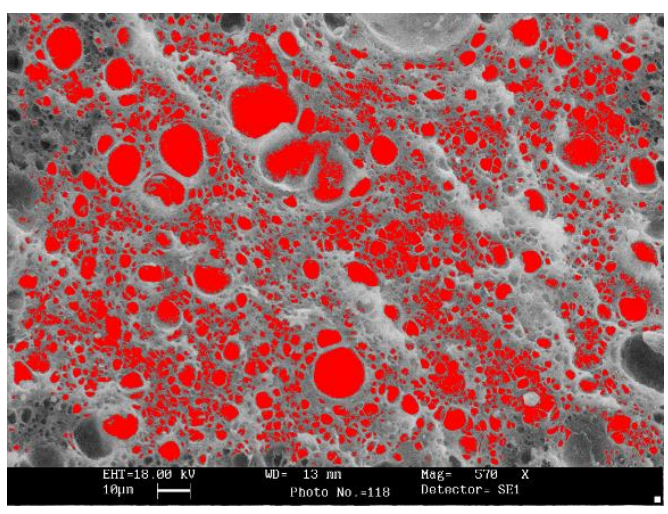

(b)

Fig. 1. Cell size measurement by image processing process a) SEM image before image processing, b) SEM image after image processing.

\section{Numerical studies}

The Least Absolute Shrinkage and Selection Operator (LASSO) is a linear regression method for regularized least-squared fits. In order to improve both the precision and generalization of regression models, the LASSO is employed for the variable selection and the regularization. The main objective of the LASSO method is to reduce the number of the model parameters by recognizing the important regressors and removing the redundant and insignificant ones. To that end, a penalty function is defined that prevents the sum of the absolute value of the regressors from increasing. This may eliminate several regressors from the model. Removing unwanted, confounding variables can also protect models against overfittings. Finally, the LASSO enhances the interpretability of the fitted models. The LASSO has advantages of both 
the stepwise selection and the ridge regression. Hence, the LASSO may result in not only more generalized and parsimonious models, but also more precise models.

Consider a dataset with $N$ input-output pairs $\left\lfloor x^{i}, y^{i}\right\rfloor$. The input has $p$ covariates as follows: $\boldsymbol{x}^{T}=\left[\begin{array}{llll}x_{1} & x_{2} & \cdots & x_{p}\end{array}\right]$

For the LASSO regression, the following cost function should be minimized:

$\min _{\beta_{0}, \boldsymbol{\beta}}\left\{\frac{1}{N} \sum_{i=1}^{N}\left(y^{i}-\beta_{0}-\boldsymbol{x}^{i^{T}} \boldsymbol{\beta}\right)^{2}\right\}$

in which $\boldsymbol{\beta}=\left[\begin{array}{llll}\beta_{1} & \beta_{2} & \cdots & \beta_{p}\end{array}\right\rfloor$.

Also, the following constraints should be considered:

$\sum_{j=1}^{p}\left|\beta_{j}\right| \leq t$

where $t$ controls the regularization. Therefore, the LASSO regression can be defined as follows:

$\min _{\beta_{0}, \boldsymbol{\beta}}\left\{\frac{1}{N} \sum_{i=1}^{N}\left(y^{i}-\beta_{0}-\boldsymbol{x}^{i^{T}} \boldsymbol{\beta}\right)^{2}+\lambda \sum_{j=1}^{p}\left|\beta_{j}\right|\right\}$

where $\lambda$ is the regularization parameter. The higher the regularization parameter, the lower the number of nonzero components.

\section{Results and discussions}

In this paper, the foam density $\rho$ and cell size A of PVM matrix foam are measured for dissimilar values of the MMT content $\Phi$, the foaming temperature $\mathrm{T}$ and the foaming time $\mathrm{t}$. Therefore, there are two dependent and three independent variables. The experimental results of the foam density $\rho$ and cell size A are illustrated in Figs. 2 and 3. 

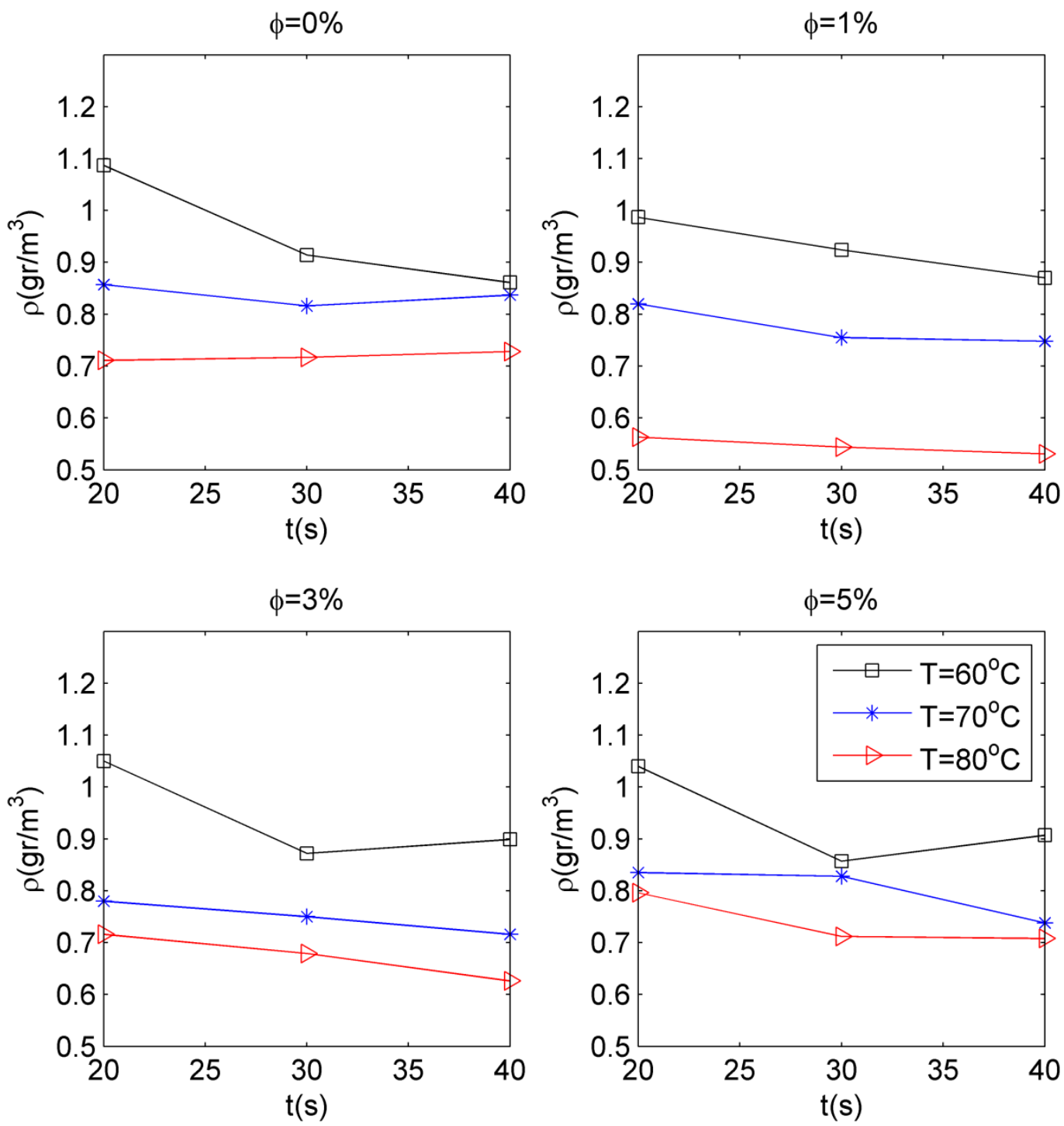

Fig. 2. The experimental results of the foam density $\rho$ at dissimilar values of the MMT content $\Phi$, the foaming temperature $\mathrm{T}$ and the foaming time t. 

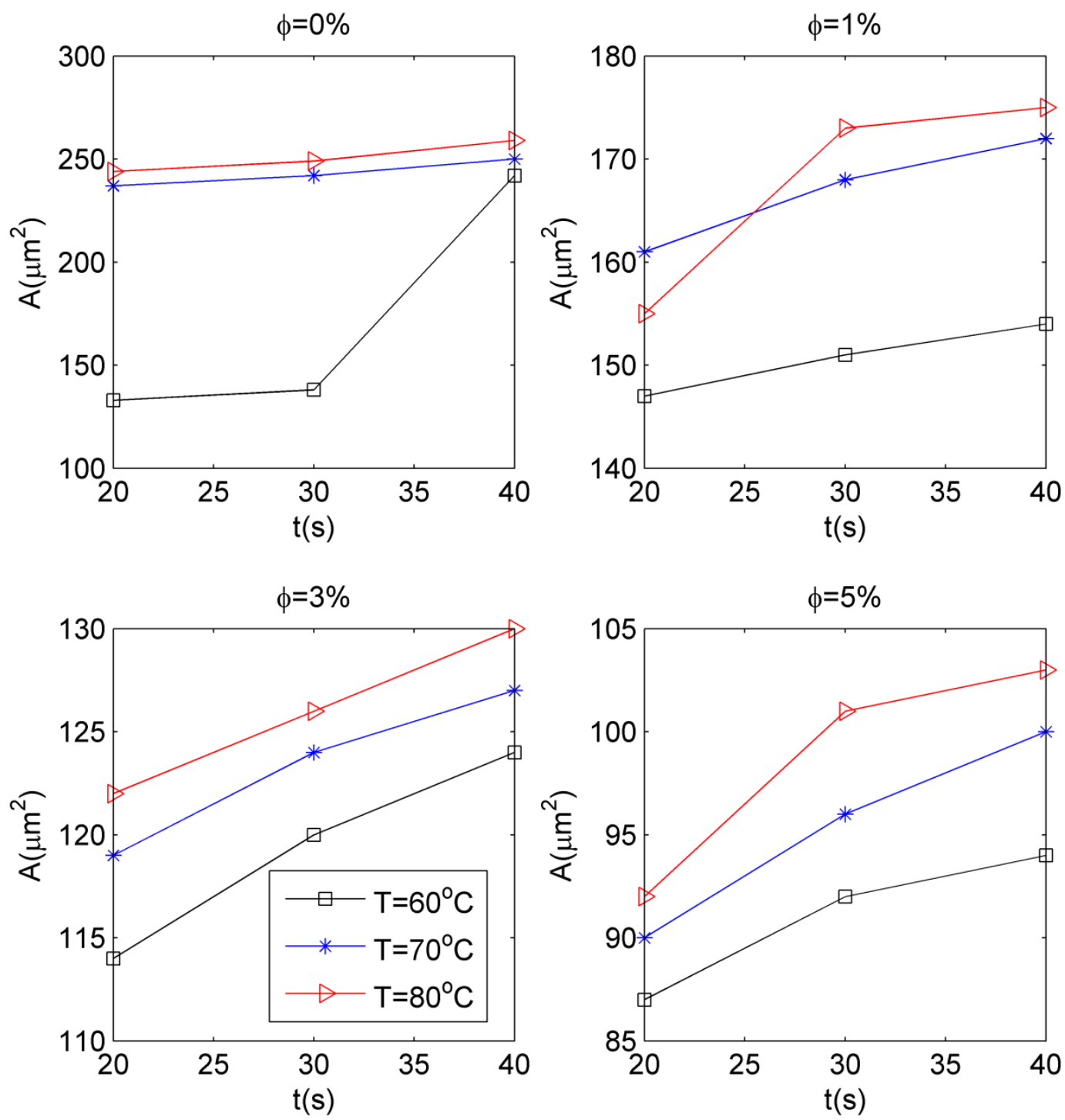

Fig. 3. The experimental results of the cell size A at dissimilar values of the MMT content $\Phi$, the foaming temperature $\mathrm{T}$ and the foaming time $\mathrm{t}$.

Now, the LASSO regression is implemented into the experimental data. To that end, a crossvalidated sequence of models with the LASSO should be obtained. The trace plots of coefficients fit by the LASSO based on the L1 norm and the regularization parameter for the foam density $\rho$ and the cell size A are illustrated in Figs. 4 and 5, respectively. The plot shows the significant coefficients in the LASSO regression for various values of $\boldsymbol{\lambda}$. The lower the value of $\lambda$, the less the regularization. The vertical lines represent $\lambda$ corresponding to minimal mean squared error. Also, the degrees of freedom (df) namely the number of nonzero 
coefficients in the regression are illustrated as a function of $\lambda$. It can be observed that there are three significant coefficients. The LASSO fits are performed by 10 -fold cross validation. Cross-validated mean squared error of the LASSO fits for the foam density $\rho$ and the cell size A are illustrated in Fig. 6.

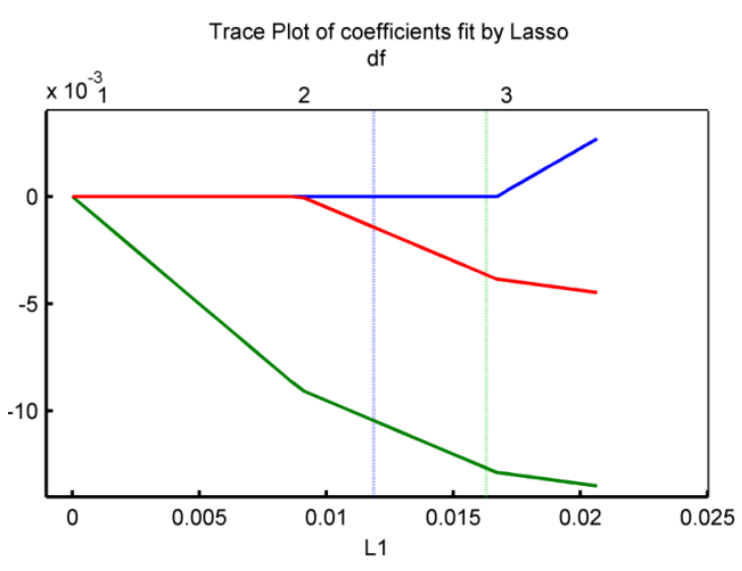

(a)

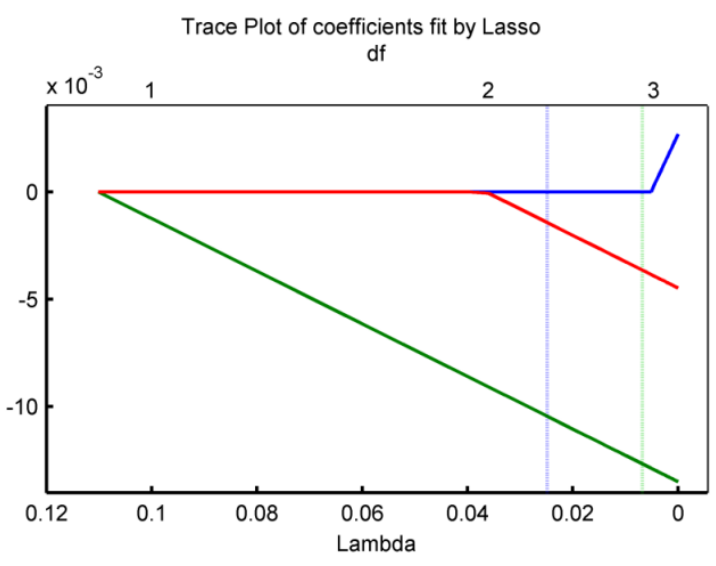

(b)

Fig. 4. Trace plot of coefficients fit for the foam density $\rho$ by the LASSO based on (a) the L1 norm and (b) the regularization parameter

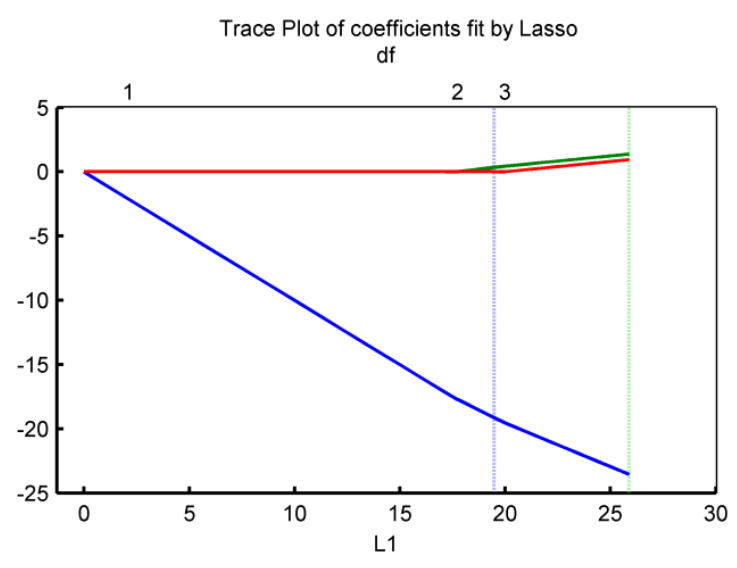

(a)

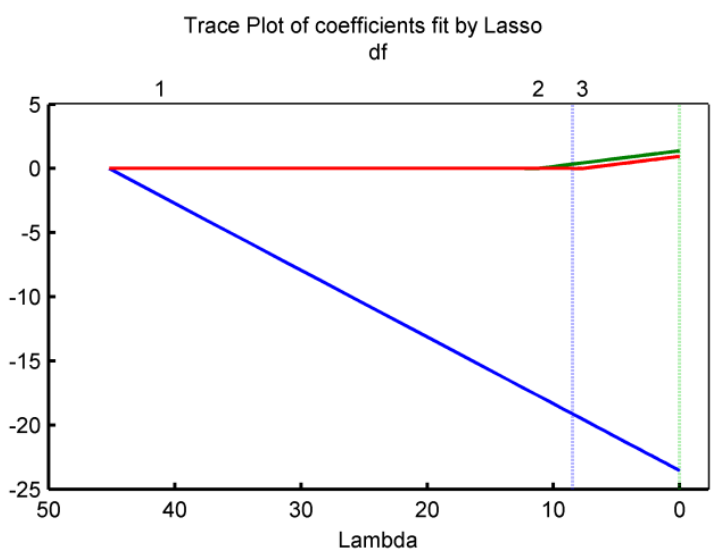

(b)

Fig. 5. Trace plot of coefficients fit for the cell size A by the LASSO based on (a) the L1 norm and (b) the regularization parameter 


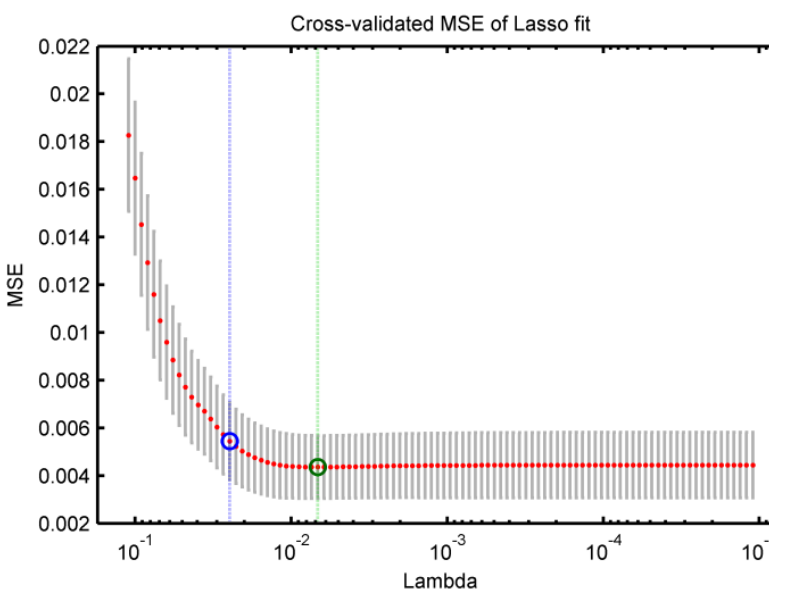

(a)

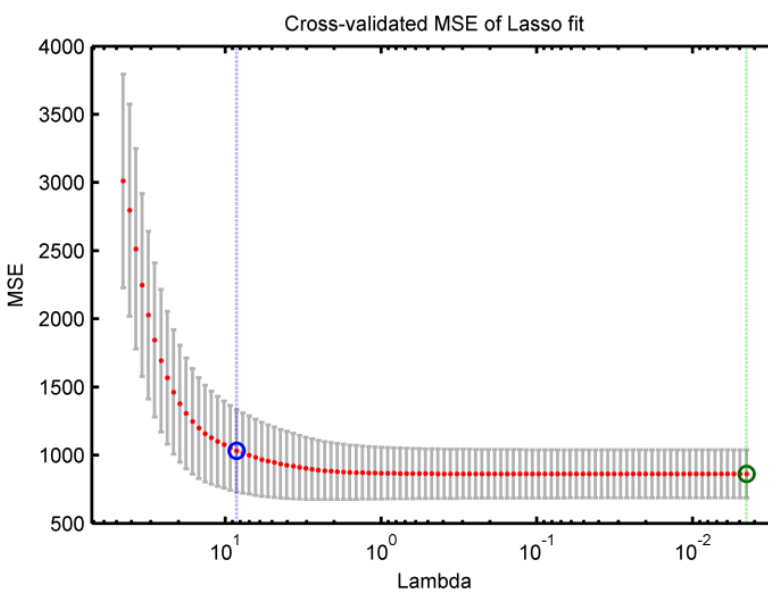

(b)

Fig. 6. Cross-Validated mean squared error of the LASSO fit for (a) the foam density and (b) the cell size.

Errors of the LASSO fit at the training dataset are depicted in Figs. 7 and 8 for the foam density and the cell size, respectively. It can be seen that the error percentages are smaller than $1.5 \%$ in all cases. Therefore, the fitted model by the LASSO regression has acceptable precision. 

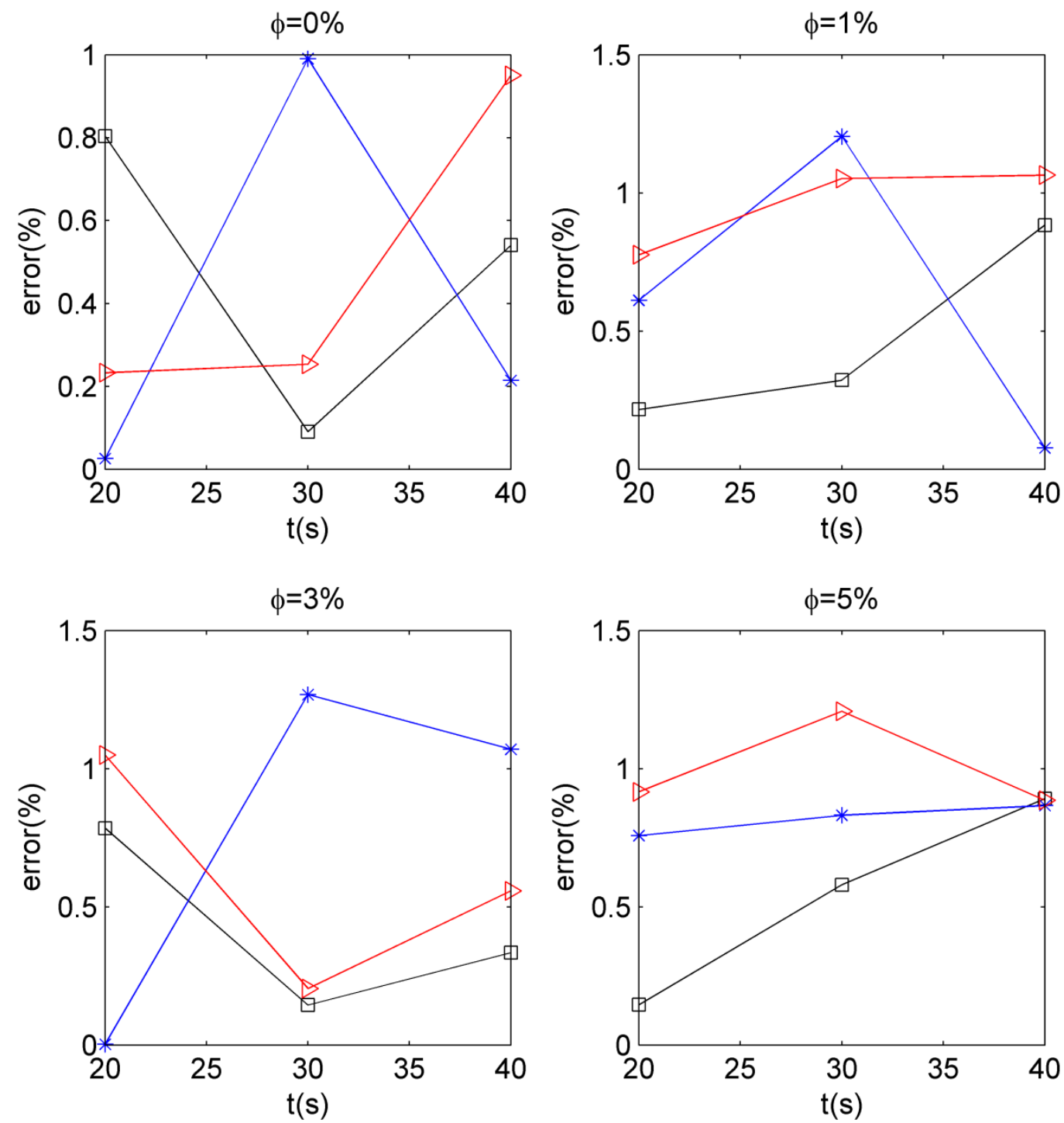

Fig. 7. Errors of the LASSO fit at the training dataset for the foam density. 

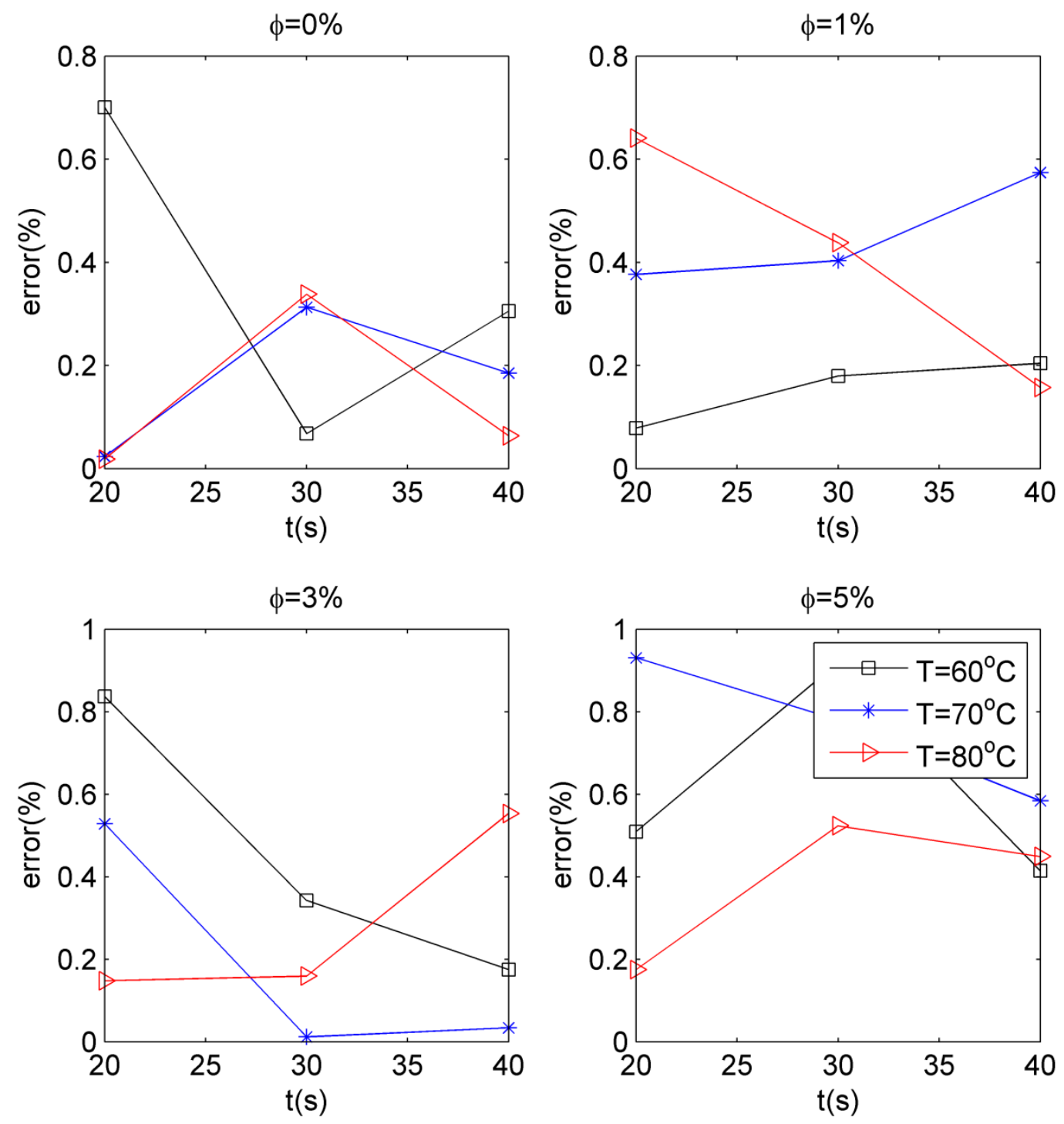

Fig. 8. Errors of the LASSO fit at the training dataset for the cell size.

Finally, the obtained LASSO models are employed in order to estimate the foam density and the cell size for all values of the MMT content, the foaming temperature and the foaming time in the examined domains. The results are illustrated in Figs. 9 and 10. Based on the results, the model has acceptable precision, continuity and smoothness. Hence, the LASSO may be an appropriate method for estimation of the sample characteristics. 


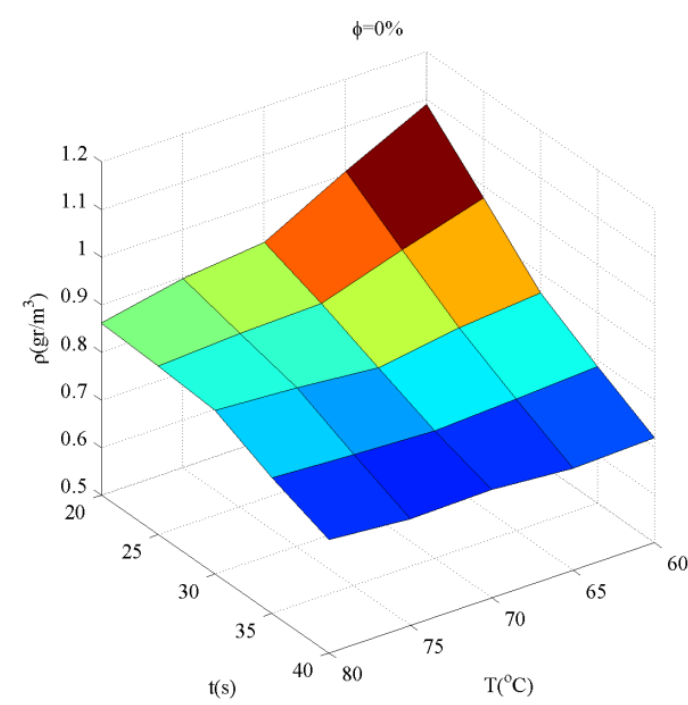

(a)

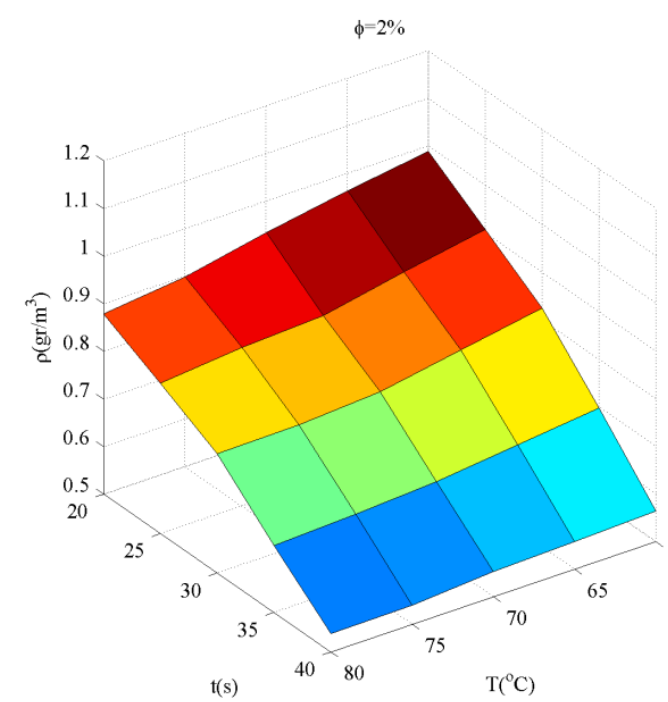

(c)
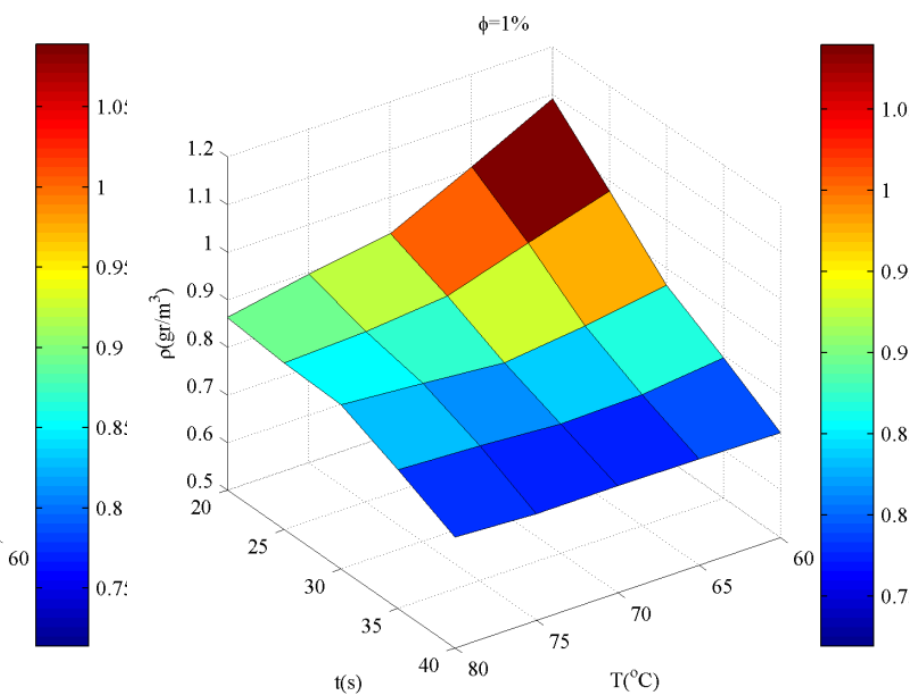

(b)
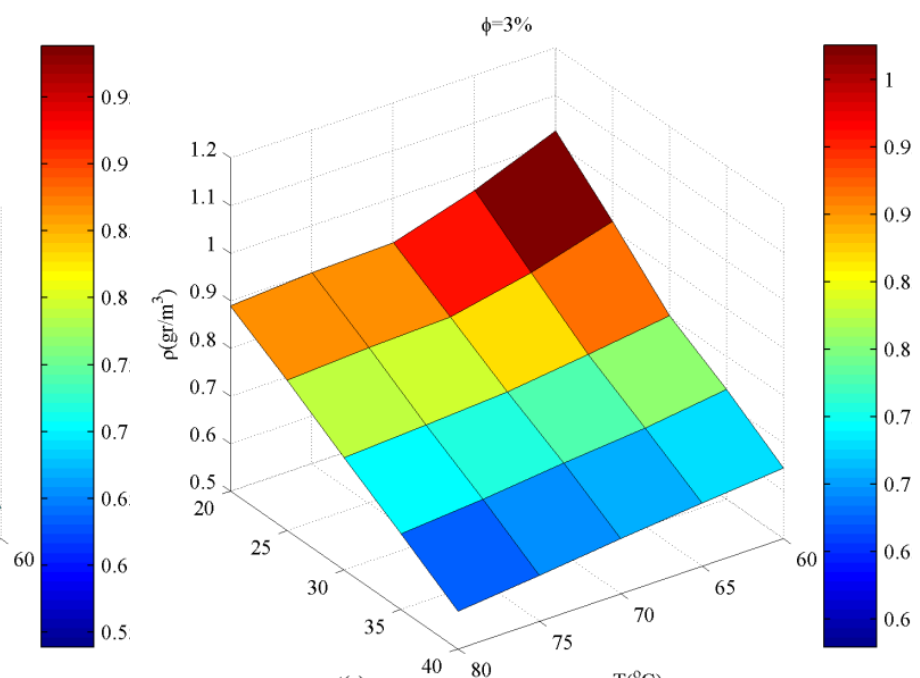

$\mathrm{t}(\mathrm{s}$

(d) 


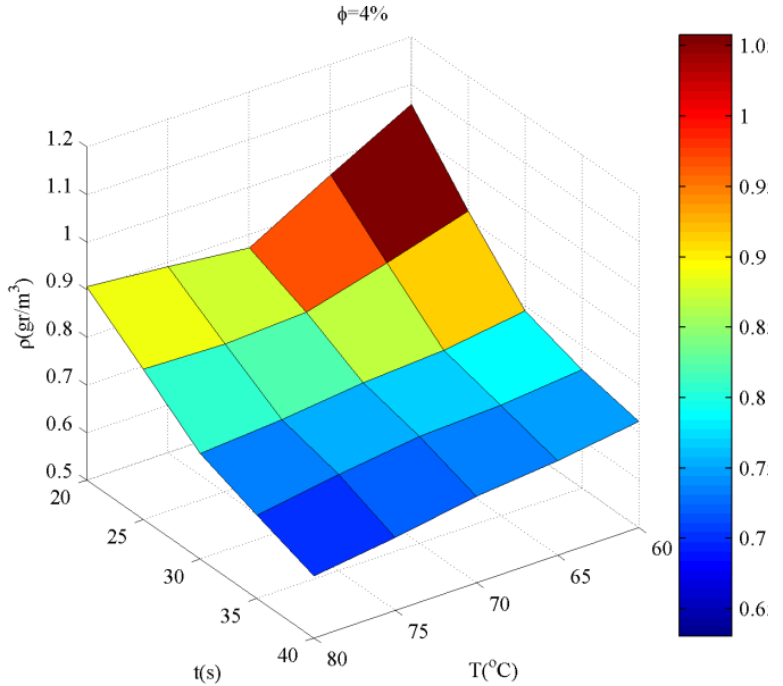

(e)

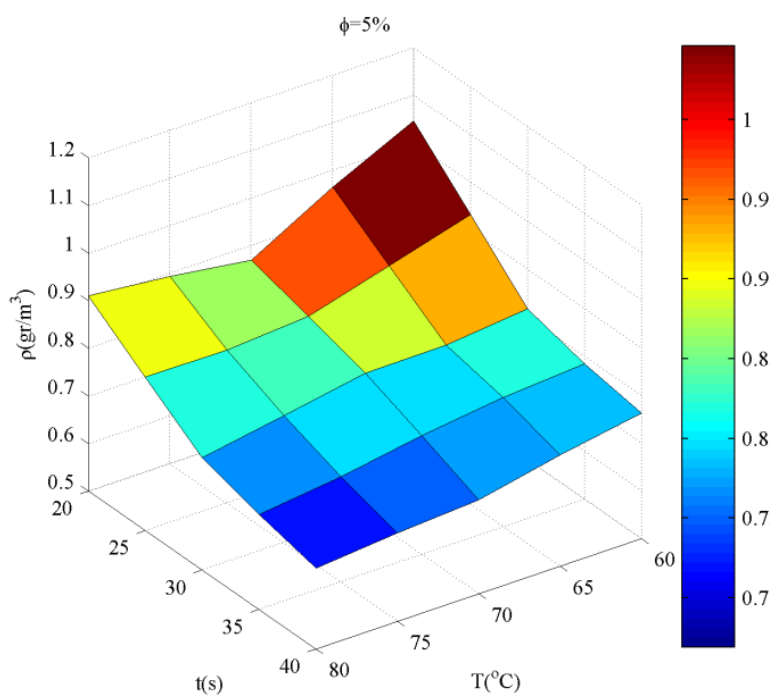

(f)

Fig. 9. The estimated foam density for all values of the foaming temperature and the foaming time in the examined domains for (a) $\phi=0 \%$, (b) $\phi=1 \%$, (c) $\phi=2 \%$, (d) $\phi=3 \%$, (e)

$$
\phi=4 \% \text { and (f) . } \phi=5 \%
$$

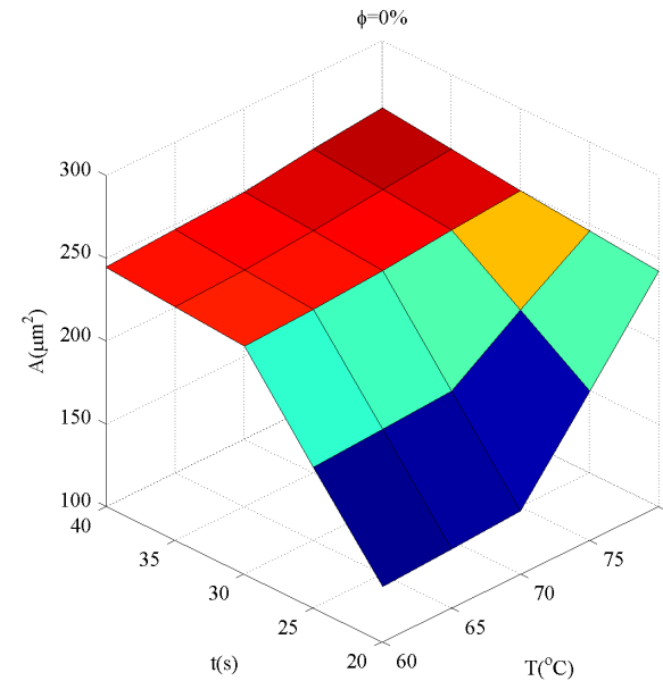

(a)
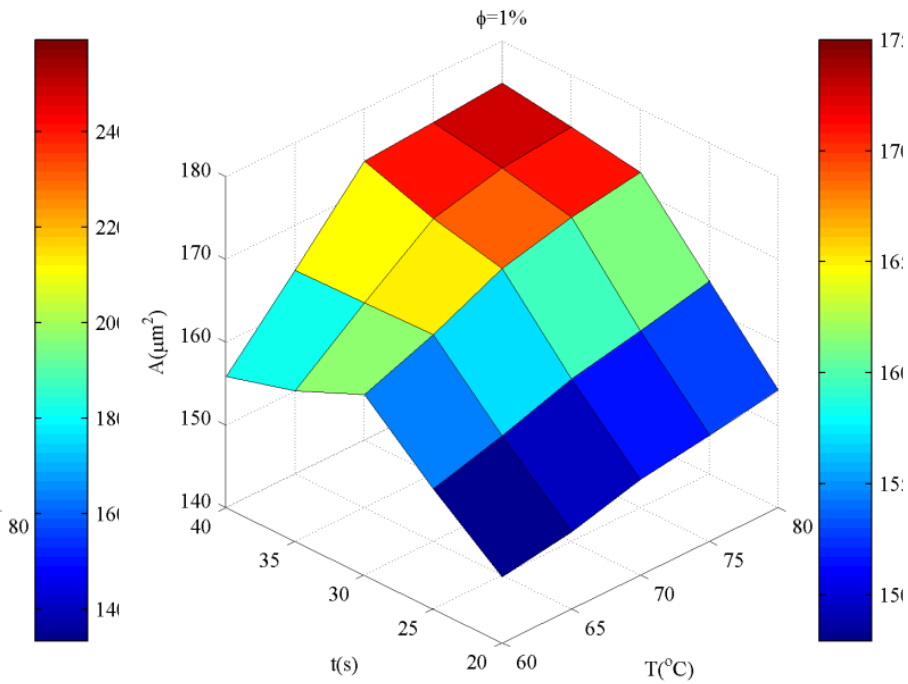

(b) 


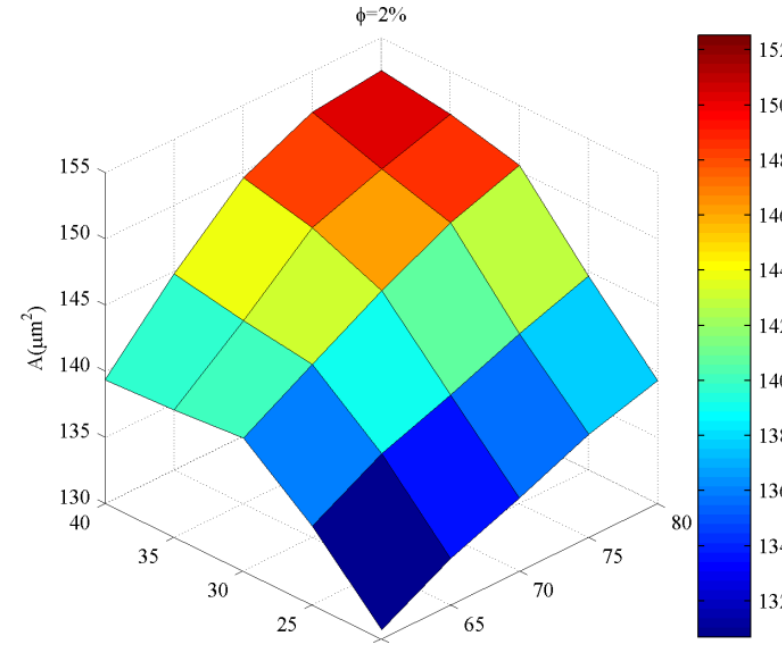

$\mathrm{t}(\mathrm{s})$ $\begin{array}{lll}20 & 60 & \mathrm{~T}\left({ }^{\circ} \mathrm{C}\right)\end{array}$

(c)

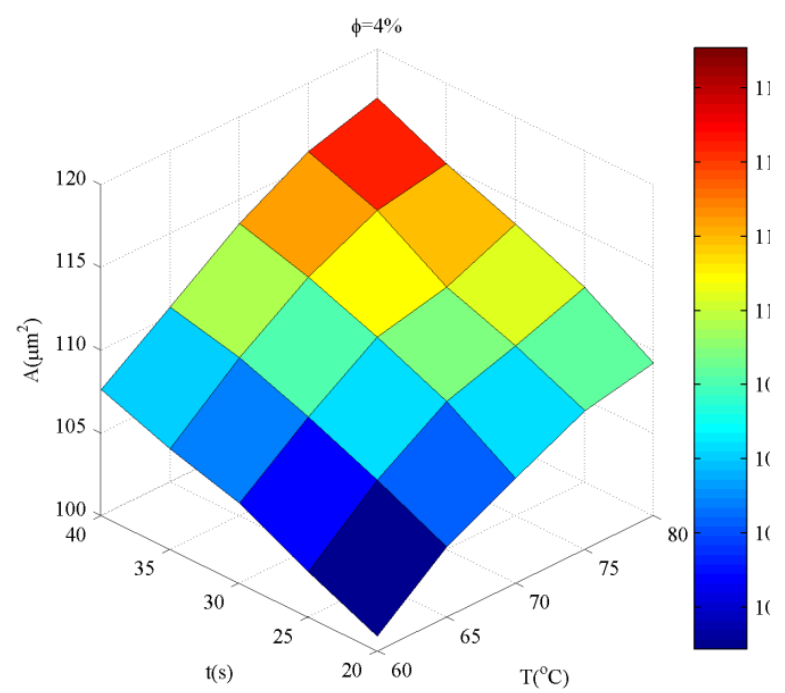

(e)

Fig. 10. The estimated the cell size for all values of the foaming temperature and the foaming time in the examined domains for (a) $\phi=0 \%$, , (b) $\phi=1 \%$, (c) $\phi=2 \%$, (d) $\phi=3 \%$, (e) $\phi=4 \%$ and (f) $\phi=5 \%$.

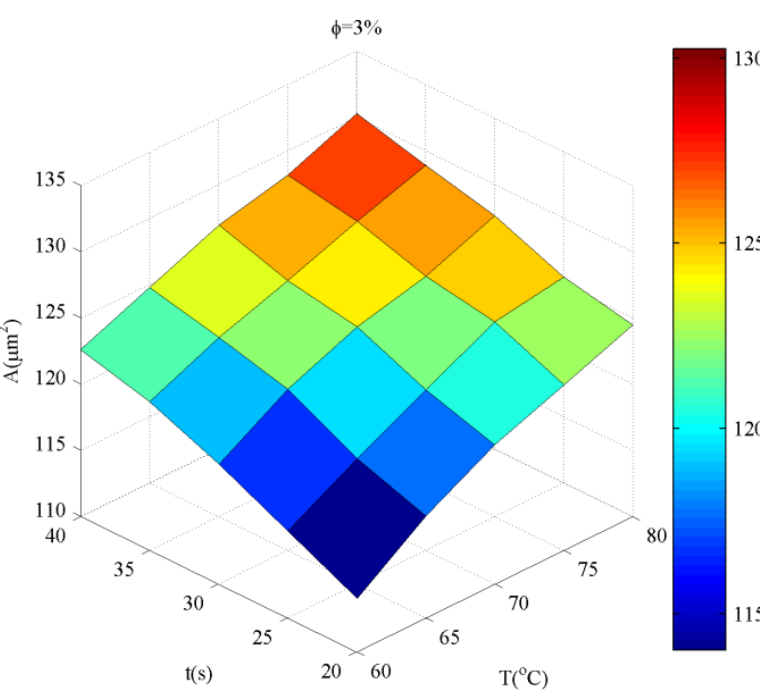

(d)

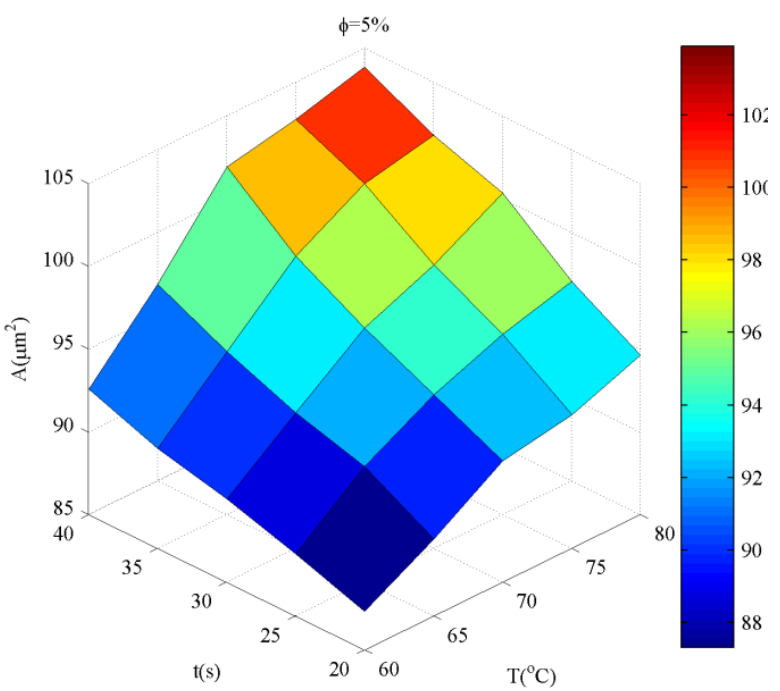

(f) 


\section{Conclusion}

The effect of nano-clay content, foaming temperature and foaming time on the density and cell size of the PVC matrix foam were studied. The cell size affected insulating and mechanical properties. Outputs consisted the density and cell size, which affected the impact of thermal conductivity, mechanical properties and the weight of the polymer foam.

Moreover the Least Absolute Shrinkage and Selection Operator (LASSO) regression method was employed in order to improve both the precision and generalization of the estimated foam density and cell size as functions of the MMT content, the foaming temperature and the foaming time.

\section{Acknowledgement:}

The first author acknowledges the support provided by NSFC (5197090691), Mindu Scholar of Minjiang University and State Key Laboratory of Hydraulic Engineering Simulation and Safety-Tianjin University (No. HESS-1901).

\section{References:}

1. W. Xu, H. Zhang, Zh. Yang, J. Zhang, "The effective thermal conductivity of threedimensional reticulated foam materials", Porous Materials, 19, 65-71 (2007).

2. I. Sumirat, Y. Ando, and S. Shimamura, "Theoretical consideration of the effect of porosity on thermal conductivity of porous materials", Porous Materials, 13, 439-443 (2006).

3. M. Ohshima, T. Nemoto, and J. Takagi, Plastics Research Online, Society of Plastics Engineers, Newtown (2010).

4. S.S. Sundarram, W. Li, "On Thermal Conductivity of Micro- and Nanocellular Polymer Foams", Polymer engineering and science, 53(9), 1901-1909 (2013).

5. M. Barmouz, A.H. Behravesh, "Statistical and experimental investigation on low density microcellular foaming of PLA-TPU/cellulose nano-fiber bio-nanocomposites", Polymer Testing, Vol. 61, 2017, Pages 300-313

6. L. M. Matuana, O. Faruk, "Effect of gas saturation conditions on the expansion ratio of microcellular poly(lactic acid)/wood-flour composites", eXPRESS Polymer Letters, Vol.4, No.10, (2010) 621-631. 
7. J.E. Martini, "The Production and Analysis of Microcellular Foam" ,Ph.D. thesis in Mechanical Engineering, Massachusetts Institude of Technology, (January.1981)

8. D. Miller, P. Chatchaisucha,V.Kumar, "Microcellular and nanocellular solid-state polyetherimide (PEI) foams using sub-critical carbon dioxide I. Processing and structure", Polymer, 50(23), 5576-5584, (2009).

9. D. Miller, V. Kumar, "Microcellular and nanocellular solid-state polyetherimide (PEI) foams using sub-critical carbon dioxide II. Tensile and impact properties", Polymer, 52(13), 29102919, (2011).

10. M. Liliana, P. Ryszard, C. Alan et al., "Processing and characterization of polyurethane nanocomposite foam reinforced with montmorillonite-carbon nanotube hybrids", Composites Part A, 44, 1-7 (2013)

11. D. Zenkert, M. Burman, "Tension, compression and shear fatigue of a closed cell polymer foam”, J. Comp. Sci. Technol. 69, 785-792 (2009)

12. A. Manninen, H. Naguib, A. Nawaby, X. Liao, M. Day, "The Effect of Clay Content on PMMA-Clay Nanocomposite Foams “, J. Cell Polym. 24, 49-70 (2005)

13. J. Reza, K. Behrad, R. Arash, "Effects of organically modified nanoclay on cellular morphology, tensile properties, and dimensional stability of flexible polyurethane foams", J. Nanostruc. Chem, 3(1), 82 (2013)

14. E. Yu, I. Manabu, O. Masami, "Foam processing and cellular structure of polylactide-based nanocomposites “, Polymer 47(15), 5350-5359 (2006)

15. Z. Changchun, N. Hossieny, Ch. Zhang, B. Wang, Sh.M. Walsh” Morphology and tensile properties of PMMA carbon nanotubes nanocomposites and nanocomposites foams", J. Compos. Sci. Technol. 82, 29-37 (2013)

16. A. Wong, S. F. L. Wijnands, T. Kuboki, Ch. B. Park, "Mechanisms of nanoclay-enhanced plastic foaming processes: effects of nanoclay intercalation and exfoliation", ournal of Nanoparticle Research,15,1815 , (2013)

17. M. Keramati, I. Ghasemi, M. Karrabi, H. Azizi, "Microcellular foaming of PP/EPDM/organoclay nanocomposites: the effect of the distribution of nanoclay on foam morphology, Polymer Journal, 44, pages 433-438 (2012). 
18. Xi.Ch. Wang, X. Jing, Y.Y. Peng, Zh, Ma, Ch.T. Liu, L.Sh. Turng, Ch.Y Shen, “The effect of nanoclay on the crystallization behavior, microcellular structure, and mechanical properties of thermoplastic polyurethane nanocomposite foams",polymer engineering and science, 65(3), 319-327 (2016)

19. H. Jadidi, H. Shahrajabian, M. Moghri, "Using the Mass Method to Produce PVC/Clay Nanocomposite Foams: The Effect of Nano-clay and Foaming Conditions on Density and Cell size”, J Inorg Organomet Polym (2016) 26:881-888

20. H, Shahrajabian, M Farahnakian, Modeling and multi-constrained optimization in drilling process of carbon fiber reinforced epoxy composite, International Journal of Precision Engineering and Manufacturing, 14(10), 2013, 1829-1837

21. M Moghri, H Shamaee, H Shahrajabian, A Ghannadzadeh, The effect of different parameters on mechanical properties of PA-6/clay nanocomposite through genetic algorithm and response surface methods, International Nano Letters, 5 (3),2015, 133-140.

22. R Azad, H Shahrajabian, Experimental study of warpage and shrinkage in injection molding of HDPE/rPET/wood composites with multiobjective optimization, Materials and Manufacturing Processes 34 (3), 2019, 274-282.

23. H Shahrajabian, M Farahnakian, Multi-constrained optimization in ball-end machining of carbon fiber-reinforced epoxy composites by PSO, Cogent Engineering, 2 (1), 2015, 993157.

24. Bagherzadeh, S.A., Sulgani, M.T., Nikkhah, V., Bahrami, M., Karimipour, A. and Jiang, Y., 2019. Minimize pressure drop and maximize heat transfer coefficient by the new proposed multi-objective optimization/statistical model composed of" ANN+ Genetic Algorithm" based on empirical data of $\mathrm{CuO}$ /paraffin nanofluid in a pipe. Physica A Statistical Mechanics and its Applications, 527, p.121056.

25. Jiang, Y., Bahrami, M., Bagherzadeh, S.A., Abdollahi, A., Sulgani, M.T., Karimipour, A., Goodarzi, M. and Bach, Q.V., 2019. Propose a new approach of fuzzy lookup table method to predict Al2O3/deionized water nanofluid thermal conductivity based on achieved empirical data. Physica A: Statistical Mechanics and its Applications, 527, p.121177.

26. Jiang, Y., Sulgani, M.T., Ranjbarzadeh, R., Karimipour, A. and Nguyen, T.K., 2019. Hybrid GMDH-type neural network to predict fluid surface tension, shear stress, dynamic 
viscosity \& sensitivity analysis based on empirical data of iron (II) oxide nanoparticles in light crude oil mixture. Physica A: Statistical Mechanics and its Applications, 526, p.120948.

27. Bagherzadeh, S.A., D’Orazio, A., Karimipour, A., Goodarzi, M. and Bach, Q.V., 2019. A novel sensitivity analysis model of EANN for F-MWCNTs-Fe3O4/EG nanofluid thermal conductivity: Outputs predicted analytically instead of numerically to more accuracy and less costs. Physica A: Statistical Mechanics and its Applications, 521, pp.406-415.

28. Karimipour, A., Bagherzadeh, S.A., Taghipour, A., Abdollahi, A. and Safaei, M.R., 2019. A novel nonlinear regression model of SVR as a substitute for ANN to predict conductivity of MWCNT-CuO/water hybrid nanofluid based on empirical data. Physica A: Statistical Mechanics and its Applications, 521, pp.89-97.

29. Bahrami, M., Akbari, M., Bagherzadeh, S.A., Karimipour, A., Afrand, M. and Goodarzi, M., 2019. Develop 24 dissimilar ANNs by suitable architectures \& training algorithms via sensitivity analysis to better statistical presentation: Measure MSEs between targets \& ANN for $\mathrm{Fe}-\mathrm{CuO} / \mathrm{Eg}-$ Water nanofluid. Physica A: Statistical Mechanics and Its Applications, 519, pp.159-168.

30. Nafchi, P.M., Karimipour, A. and Afrand, M., 2019. The evaluation on a new nonNewtonian hybrid mixture composed of $\mathrm{TiO} 2 / \mathrm{ZnO} / \mathrm{EG}$ to present a statistical approach of power law for its rheological and thermal properties. Physica A: Statistical Mechanics and its Applications, 516, pp.1-18.

31. Dehghani, Y., Abdollahi, A. and Karimipour, A., 2019. Experimental investigation toward obtaining a new correlation for viscosity of WO 3 and Al 2 O 3 nanoparticles-loaded nanofluid within aqueous and non-aqueous basefluids. Journal of Thermal Analysis and Calorimetry, 135(1), pp.713-728.

32. Alrashed, A.A., Karimipour, A., Bagherzadeh, S.A., Safaei, M.R. and Afrand, M., 2018. Electro-and thermophysical properties of water-based nanofluids containing copper ferrite nanoparticles coated with silica: experimental data, modeling through enhanced ANN and curve fitting. International Journal of Heat and Mass Transfer, 127, pp.925-935.

33. Karimipour, A., Bagherzadeh, S.A., Goodarzi, M., Alnaqi, A.A., Bahiraei, M., Safaei, M.R. and Shadloo, M.S., 2018. Synthesized $\mathrm{CuFe} 2 \mathrm{O} 4 / \mathrm{SiO} 2$ nanocomposites added to water/EG: Evaluation of the thermophysical properties beside sensitivity analysis \& EANN. International Journal of Heat and Mass Transfer, 127, pp.1169-1179. 
